\title{
Recurrence after correction of acquired ankle equinus deformity in children using Ilizarov technique
}

\author{
Khaled M. Emara • Mohamed Farouk Allam • \\ Mohamed Nabil M. A. ElSayed • \\ Khaled Abd E. L. Ghafar
}

Received: 6 May 2008/Accepted: 4 November 2008/Published online: 20 November 2008

(C) Springer-Verlag 2008

\begin{abstract}
To describe our Ilizarov technique for the treatment of acquired equinus deformity in children and to determine if compliance with continuous use of an ankle foot orthosis (after removal of the fixator and until skeletal maturity) can influence the severity of recurrence. A cohort of 26 children with post-traumatic or post-burn contractures producing an equinus deformity was followed up for a minimum of 2 years after skeletal maturity. Cases with a bony deformity and/or nerve injury were excluded from this study. All patients were managed by a percutaneous tendo-Achilles lengthening followed by application of an Ilizarov external fixator. Post-operative treatment was in the form of gradual correction at a rate of $0.5 \mathrm{~mm}$ per day. Correction started from the second postoperative day until an over-correction of 15 degrees dorsiflexion was achieved. Ankle range of movement was encouraged 4 weeks prior to removal of the external fixator. On removal of the fixator, a posterior splint was applied until substituted by an ankle foot orthoses (AFO). The AFO was used continuously during the first 2-3 months and at nighttime thereafter until skeletal maturity. Fifteen children were compliant with the use of the AFO until skeletal maturity and 11 non-compliant. We compared the recurrence and the size of
\end{abstract}

K. M. Emara $(\bowtie) \cdot$ M. N. M. A. ElSayed · K. A. E. L. Ghafar Department of Orthopedic Surgery,

Ain Shams University Hospitals,

13 B Kornesh El Nile, Agha Khan,

Cairo, Egypt

e-mail: kmemara@hotmail.com

M. F. Allam

Department of Community, Environmental and Occupational

Medicine, Faculty of Medicine, Ain Shams University,

Cairo, Egypt deformity between the two groups. The rate of recurrence, degree of equinus at recurrence and number of episodes of external fixation surgery showed statistical significant differences $(P<0.01)$ between the groups. The Ilizarov technique for treatment of acquired equinus deformity secondary to soft tissue scarring is a safe and effective technique. The use of an AFO until skeletal maturity can decrease the risk and degree of recurrence.

Keywords Equinus - Ilizarov - External fixation · Ankle · Deformity

\section{Introduction}

In children, an acquired equinus deformity can affect the ankle or forefoot (plantaris) and is due to many etiologies. This includes post-traumatic and post-burn soft tissue scarring, neurological conditions as cerebral palsy and poliomyelitis, muscle scarring after compartment syndrome, prolonged immobilization in plantarflexion, as a complication of limb lengthening and other etiologies [1].

Prevention is achieved through avoiding the predisposing factors, physical therapy and splinting. In cases with established equinus, treatment includes physical therapy, manipulation and casting in mild cases [2, 3]. In severe cases, this may need a soft tissue release by tendo-Achilles lengthening, with or without a posterior ankle capsulotomy, and lengthening of other contracted soft tissues including the skin by microsurgical soft tissue transfer [4, 5]. Distraction histogenesis using the Ilizarov technique is another safe and effective line of treatment for such complex problem [6].

In the treatment of an acquired equinus deformity in children, the main complication encountered with all the 
previously cited techniques is a high risk of recurrence [6-8]. This may be due to what some authors call 'soft tissue memory'. Another cause may be the discrepancy between the rate of growth of the bone and the contracted soft tissues [6,9]. In order to avoid or decrease the problem of recurrence, some authors recommended bony fusion when it is feasible and the child is of an appropriate age while others recommended continuous splinting until skeletal maturity [10].

In this study, we present our surgical technique in the treatment of acquired equinus in children, without associated neurological deficit, using distraction histogenesis through external fixation. The aim of the study is to determine if compliance with an AFO after removal of external fixation can prevent the degree of recurrence.

\section{Materials and methods}

Fifty-six cases with equinus deformity in skeletally immature patients were referred for treatment by the Ilizarov technique between the year 2000 and 2004. Twenty-six were post-traumatic or post-burn contractures with a minimum follow-up of 2 years after skeletal maturity. The inclusion criteria included soft tissue contractures without associated bone deformity or nerve injury (Fig. 1). Exclusion criteria were skeletally mature patients at first presentation, polio and cerebral palsy patients, associated bone deformity or joint destruction, epiphyseal injuries causing deformity and open or infected wounds. Initial assessment included a history and physical examination together with plain radiographs of both ankles and feet to exclude associated bone deformity $[1,11]$.

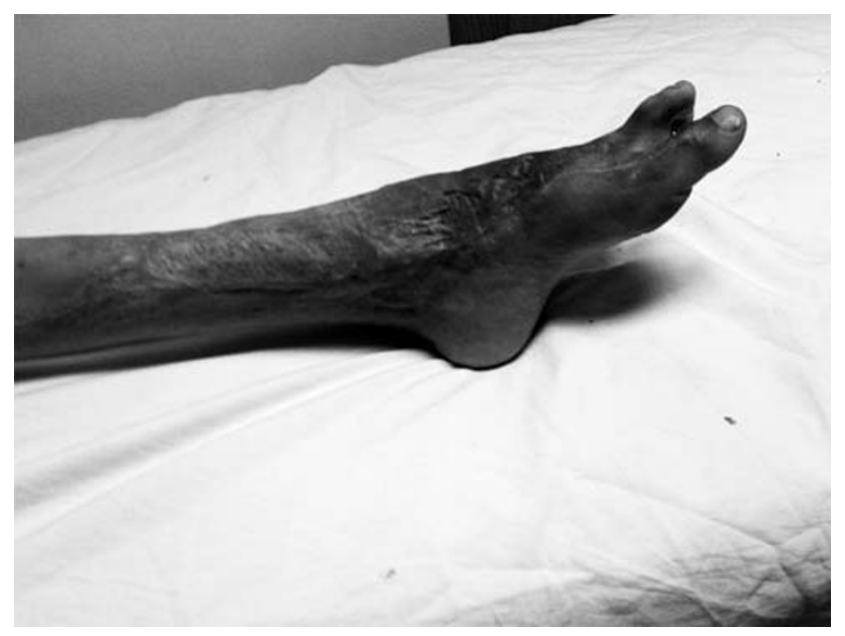

Fig. 1 A case of 10-year-old boy with post traumatic soft tissue loss managed by multiple surgeries with secondary fixed equines due to soft tissue scarring
Technique

Surgical management under general anesthesia was a percutaneous tendo-Achilles lengthening followed by application of an Ilizarov external fixator. The frame was composed of two rings attached to the tibia and a $5 / 8$ ring attached to the hind foot and another attached to the forefoot. The frame used to correct the ankle equinus was unconstrained; no hinges were applied at the axis of rotation of the ankle joint and correction axis was determined by the ankle joint itself. Correction forces were in the form of two distraction rods posterior to the axis of the ankle joint and two compression rods anterior to the axis of the ankle. The use of two rods instead of one was to correct associated varus or valgus by a differential rate of distraction between the rods. The use of posterior distraction rods instead of only anterior compression ones was to avoid compressive forces on the ankle joint cartilage and the distal tibial physis. Another distraction rod was placed between the hind foot and forefoot $5 / 8$ rings on the medial side to correct associated cavus that develops during correction. Kirschner-wire transfixation of the big toe interphalangeal and metatarso-phalangeal joints was performed to avoid joint subluxation or dislocation during equinus correction.

Post-operative treatment was in the form of gradual correction at a rate of $0.5 \mathrm{~mm}$ per day. The correction started from the second post-operative day until a position of overcorrection of 15 degrees dorsiflexion at the ankle was achieved (Fig. 2). Thereafter the external fixator was kept in situ for twice the period that was needed to achieve the correction. At 4 weeks prior to removal of external fixation, ankle range of movement exercises was prescribed by temporary removal of the distraction and compression rods for $1-2 \mathrm{~h}$ daily, under supervision of a physical therapist or

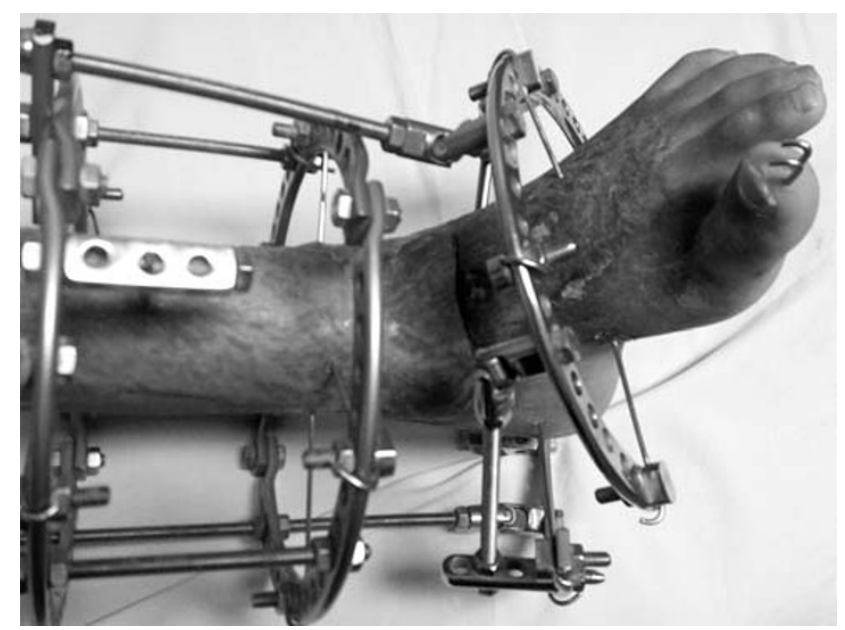

Fig. 2 This figure is showing the same patient in Fig. 1 during treatment by Ilizarov external fixation and gradual distraction histogenesis and associated pinning of the big toe and first metatarso-phalangeal joint 
the family, with regular manipulations of the ankle and toe joints simultaneously. Removal of external fixation was done without anesthesia and measurement for an ankle foot orthosis (AFO) performed immediately. A posterior splint was applied until the AFO was manufactured and fitted.

The AFO is used for $24 \mathrm{~h}$ every day during the first 2-3 months and then at nighttime until skeletal maturity. The use of the AFO was discontinued when the patient reached skeletal maturity. Not all patients were compliant with the instruction to use the AFO due to social factors. Of the 26 patients, 15 were compliant with the use of the AFO until skeletal maturity and 11 were non-compliant. We compare the risk of recurrence and the degree of recurrence between both groups of patients.

\section{Statistical analysis}

Comparisons between ankle splinting using the AFO for a short period after removal of Ilizarov external fixation and continuous use of the AFO until skeletal maturity were done using Mann-Whitney $U$ test for continuous variables and Fisher's Exact test for categorical variables.

Data entry and statistical analysis were done using statistical package for social science (SPSS) version 12.0. Level of significance was set at a $P$ value $\leq 0.05$.

\section{Results}

Of the 26 patients included in our study, there were 9 (34.6\%) females and $17(65.4 \%)$ males. The mean age at injury (trauma or burns) was $7.6 \pm 2$ years (range 4-11). The pre-operative equinus deformity ranged from 50 to 70 degrees with mean of 60.4 degrees.

Four (15.4\%) patients had bilateral equinus. Of the remaining 21 patients, $9(34.6 \%)$ had equinus of the right ankle and $13(50 \%)$ on the left side. In $5(19.2 \%)$ patients, both equinus and knee flexion deformities were present. The causes of equinus deformities were burn contractures $(46.2 \%)$ and soft tissue loss after motor vehicle accidents $(53.8 \%)$.

The mean age at surgery was $10.9 \pm 1.3$ years (range $8-13)$. The mean time in the fixator was $8.2 \pm 1.4$ months (range 6-11). All patients had their deformities fully corrected. The follow-up duration ranged from 3 to 7 years with mean of 5.5 years (SD 1.2). At the final follow-up, 5 $(19.2 \%)$ patients required another episode of treatment by external fixation for recurrence of the equinus (all were from the group of AFO non-compliant patients).

Eighteen patients $(69.2 \%)$ had recurrent equinus and only $8(30.8 \%)$ had no relapse. The severity of recurrent deformity varied widely and ranged from 5 to 40 degrees.

Comparisons of the two groups with regard to age at injury in years, age at fixation in years, sex, side of equinus, deformity type, cause of equinus, size of pre-operative deformity, time in fixator in months and follow-up duration were not significant different statistically. In contrast, presence of a recurrence, time to recurrence in months, degree of equinus at recurrence and number of treatments by external fixation were statistically different $(P<0.01)$ with the exception for time to recurrence (Table 1) (Fig. 3).

Table 1 The comparison between ankle splinting using AFO after removal of Ilizarov external fixation and continuous use of AFO till skeletal maturity

\begin{tabular}{|c|c|c|c|}
\hline Variables & $\begin{array}{l}\text { Ankle foot orthosis } \\
\text { only after removal of } \\
\text { Ilizarov external }(N=11)\end{array}$ & $\begin{array}{l}\text { Continuous use of ankle } \\
\text { foot Orthosis till skeletal } \\
\text { maturity fixation }(N=15)\end{array}$ & $P$ value $^{\#}$ \\
\hline Age at injury in years & $7.4 \pm 1.9$ & $7.9 \pm 2.1$ & 0.48 \\
\hline Age at fixation in years & $10.6 \pm 1.3$ & $11.1 \pm 1.3$ & 0.17 \\
\hline Sex (male) & $7(63.6)$ & $10(66.7)$ & 0.87 \\
\hline Side (bilateral) & $2(18.2)$ & $2(13.3)$ & 0.91 \\
\hline Deformity (equinus) & $9(81.8)$ & $12(80)$ & 0.91 \\
\hline Cause of equinus (burn) & $5(45.5)$ & $7(46.7)$ & 0.95 \\
\hline Pre-operative equinus degree & $60.9 \pm 8.3$ & $60 \pm 8.4$ & 0.78 \\
\hline Time in fixator in months & $8.2+0.98$ & $8.3 \pm 1.3$ & 0.89 \\
\hline Follow up duration in years & $5.5 \pm 1.2$ & $5.6 \pm 1.2$ & 0.79 \\
\hline Recurrence & $11(100)$ & $7(46.7)$ & 0.007 \\
\hline Time to recurrence in months & $26.6 \pm 12.9$ & $24.5 \pm 28.3$ & 0.60 \\
\hline Degree of equinus at recurrence & $21.8 \pm 10.3$ & $3 \pm 3.7$ & 0.000 \\
\hline Number of external fixation surgery $(>1)$ & $5(45.5)$ & $0(0)$ & 0.007 \\
\hline
\end{tabular}

Quantitative data are expressed as mean \pm SD; Number in parentheses adjacent to the actual number indicates percentage of cases

\# Mann-Whitney $U$ test for continuous variables and Fisher's Exact test for categorical variables 


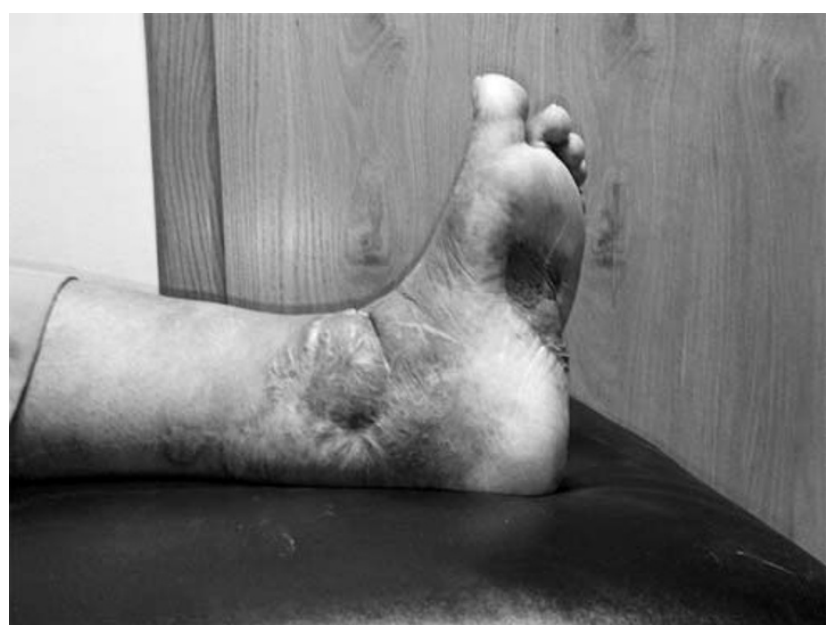

Fig. 3 The patient at age of 18 with no recurrence of equines

\section{Discussion}

The Ilizarov technique in the management of equinus from soft tissue scarring is effective and safe. Other techniques of soft tissue release need an extensile approach that is associated with high risk of wound complications due to the poor vascularity of the skin and foot [12]. A major problem encountered with such techniques is the recurrence of deformity. The rate of recurrence after correction of equinus deformity in children using the Ilizarov technique ranges from 34 to $76 \%[6-9,12]$. Some studies recommended bony fusion as early as possible in the management of such cases to avoid recurrence [10] but early ankle fusion is associated with a risk of limb length discrepancy or deformity if the distal tibial physis is injured, a loss of ankle movement and abnormal stresses on the growing limb $[9,10]$.

Carmichael et al. [6] reviewed 23 cases of post-burn ankle equinus treated by the Ilizarov technique of gradual distraction histogenesis. A full correction was achieved in all patients but the recurrence rate was $74 \%$ (17/23). The average degree of ankle equinus 34 degrees (34 degrees of plantarflexion) before surgery corrected to +7 degrees $(7$ degrees of dorsiflexion) after treatment. In this study, the average degree of ankle equinus preoperatively was 60.4 degrees. Similarly, the average age at the time of index procedure $10.9 \pm 1.3$ years compared to 9.4 years in the previous study. At final follow up, the average degree of equinus in the compliant group of patients was $3 \pm 3.7$ degrees and in non-compliant patients $21.8 \pm 10.3$ degrees; this compares to 25 degrees (range 5-75) in the other study.

It is necessary to consider a number of limitations in this study. A potential source of bias is the small sample size. A randomized controlled clinical trial comparing an intervention technique with 11 versus 15 patients has limited external validity. We used nonparametric statistical tests to minimize the probability of false significant results due to the small sample size. Additionally, the postoperative results were compared using qualitative and quantitative analyses (recurrence, time to recurrence and degree of equinus at recurrence) to confirm a difference in the effect of compliance with the AFO.

\section{Conclusion}

We conclude that use of an AFO helps to decrease the risk and the degree of recurrence in such group of patients. Continuous use of AFO until skeletal maturity had a lower recurrence rate and smaller degree of equinus at recurrence compared to those with a limited period of AFO use after removal of Ilizarov external fixation. Close follow-up is needed and the possible need for further surgery needs to be explained to the patient and the family.

\section{References}

1. Digiovanni CW, Holt S, Czerniecki JM, Ledoux WR, Sangeorzan BJ (2001) Can the presence of equinus contracture be established by physical exam alone? J Rehabil Res Dev 38(3):335-340

2. Dursun E, Dursun N, Alican D (2002) Ankle-foot orthoses: effect on gait in children with cerebral palsy. Disabil Rehabil 24(7):345-347

3. Sobel E, Levitz SJ, Caselli MA (1999) Orthoses in the treatment of rearfoot problems. J Am Pediatr Med Assoc 89(5):220-233

4. Segev E, Wientroub S (2007) A combined use of a free vascularised flap and an external fixator for reconstruction of lower extremity defects in children. J Orthop Surg 15(2):207-210

5. Serletti JM, Schingo VA Jr, Deuber MA, Carras AJ, Herrera HR, Reale VF (1996) Free tissue transfer in pediatric patients. Ann Plast Surg 36(6):561-568

6. Carmichael KD, Maxwell SC, Calhoun JH (2005) Recurrence rates of burn contracture ankle equinus and other foot deformities in children treated with Ilizarov fixation. J Pediatr Orthop 25:523-528

7. Hahn SB, Park HJ, Park HW, Kang HJ, Cho JH (2001) Treatment of severe equinus deformity associated with extensive scarring of the leg. Clin Orthop Relat Res 393:250-7

8. Huang SC (1996) Soft tissue contractures of the knee or ankle treated by the Ilizarov technique. High recurrence rate in 26 patients followed for 3-6 years. Acta Orthop Scand 67:443-449

9. Guan WX (1985) A new method for treating postburn talipes equinovarus. Ann Plast Surg 15(6):515-518

10. Steinwender G, Saraph V, Zwick EB, Uitz C, Linhart W (2001) Complex foot deformities associated with soft-tissue scarring in children. J Foot Ankle Surg 40(1):42-49

11. Elomrani N, Kasis A, Saleh M (2005) A radiographic technique for the assessment of ankle and midfoot equinus. Foot Ankle Int 26(3):251-255

12. Melvin JS, Dahners LE (2006) A technique for correction of equinus contracture using a wire fixator and elastic tension. J Orthop Trauma 20(2):138-142 